\title{
Analysis of the Mechanical Self-noise Transmission of Underwater Acoustic Field on the Sonar
}

\author{
Daokun Zhang \\ School of Mechanical Engineering, Shandong \\ University, \\ Key Laboratory of High Efficiency and Clean \\ Mechanical Manufacture, Shandong University, \\ Jinan, China \\ E-mail: zhangdaokun1030@163.com
}

Rui Huo

School of Mechanical Engineering, Shandong

University,

Key Laboratory of High Efficiency and Clean

Mechanical Manufacture, Shandong University, Jinan, China

E-mail: huorui@sdu.edu.cn

\author{
Zhidong Wang \\ School of Mechanical Engineering, Shandong \\ University, \\ Key Laboratory of High Efficiency and Clean \\ Mechanical Manufacture, Shandong University, \\ Jinan , China \\ E-mail:wzdhbdjk@163.com
}

\begin{abstract}
Combining with the structure characteristics of sonar cavity and the theory of the self-noise prediction, the wall of sonar platform affected by the mechanical vibration is simulated by elastic thin plate and the rectangular cavity is the simulation of sonar cavity, the theoretical analysis model of the sonar cavity is established considering the fluidstructure coupling effect. The acoustic field in sonar cavity is analyzed theoretically using the modal methods, and the distribution of sound pressure in acoustic field is obtained when the elastic boundary is motivated externally. Underwater acoustic field of the rectangular cavity is analyzed through the numerical examples by the MATLAB, the change rule of the acoustic field in sonar cavity is studied. The finite element analysis is carried out on the rectangular cavity model by acoustic software of the LMS Virtual. lab in order to check the correctness of the theoretical model. This study provides certain theoretical basis for the analysis and control of mechanical self-noise sonar cavity at the low frequency.
\end{abstract}

Keywords-sonar cavity; mechanical noise; rectangular cavity; fluid solid coupling; acoustic field

\section{INTRODUCTION}

Sonar is a kind of water detection equipment widely used in ships and submarines [1]. It is the key to improve sonar performance that improves the detection performance of sonar and the stealthiness of the ship [2]. The working principle of sonar is to use propagation characteristics of sound waves in the water to probe, communicate, identify and guide, etc. Its main performance indicators include the maximum and minimum range, resolution, transmission frequency, directional accuracy, receiving sensitivity, etc.[5]. Sonar detection ability does not only depend on the technical performance of the sonar equipment itself, but also the size of its background noise in the work environment. The selfnoise on the sonar refers to noise not from the detection target that all kinds of environmental noise along with the vessel sailing. The self-noise includes four noise component of the machinery noise, propeller noise, hydrodynamic noise and the marine environment noise according to the noise source on the sonar in the process of sailing ships [9]. Generally it is hard to control of the marine environmental noise and it is very small compared with the level of overall noise, so the ocean ambient noise is neglected commonly [11]. The mechanical noise at the working of mechanical equipment contributes to the noise in the low speed and contributes to the noise less in high speed. When the ship speed is low the existence of mechanical self-noise affects the quiet of the sonar cavity and make great influence on the concealment of the ships [12]. The main influence factors can be determined through the analysis of the mechanical self-noise of sonar; it can have profound research significance for the control of the mechanical self-noise of sonar and the improvement of detecting ability at the low frequency $[13,14]$.

\section{THE THEORY ANALYSIS MODEL OF SONAR CAVITY CONSIDERING FLUID-STRUCTURE COUPLING EFFECT}

A. The establishment of the model

Fig.1 shows the model description about the working space of sonar array in the sonar dome. Usually working space of sonar array is irregular, it is assumed that there is structural acoustic excitation in some areas of the plane and the acoustic field will be formed in the sonar cavity by the excitation that is known as the source of mechanical self-noise. 
Acoustic field of sonar cavity meets the wave equation

$$
\nabla^{2} p-\left(1 / \mathrm{c}_{0}^{2}\right) \partial^{2} p / \partial^{2} t=0
$$

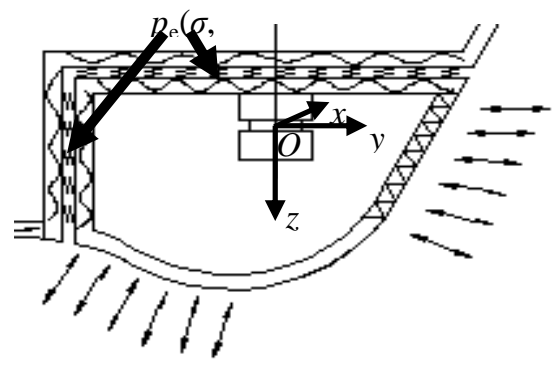

Figure 1. Theory model of the sonar cavity

Where $p$ is the sounds pressure and $\mathrm{c} 0$ is the wave velocity. In the boundary of the sonar $\mathrm{D}$, if the border is not rigid, when

$$
\partial p / \partial \boldsymbol{n}=-\rho_{0} a_{n}(\sigma, t)
$$

Where $n$ is the outside normal direction at the border of cavity wall, $\rho_{0}$ is the density of the medium (water), $a_{n}$ is the normal vibration acceleration of the cavity wall. If there is the area of rigid cavity wall Ds, the boundary conditions is $\partial p / \partial \boldsymbol{n}=0$.

\section{B. $\quad$ Acoustic mode equation}

If the borders are all rigid (Ds=D), equation (1) has the modal solution like the form of $F_{r}(\sigma) e^{j \omega t}(\mathrm{r}=0,1,2, \ldots)$, and in order to solve the wave equation through the modal method, the following differential equations are obtained by citing gauss formula transform.

$$
\stackrel{\mathbb{W}}{P}_{r}+\left(\omega_{r}^{A}\right)^{2} P_{r}=-A_{r} / V(\mathrm{r}=0,1,2 \ldots)
$$

Where

$$
A_{r}=\iint_{D} F_{r} a_{n} d A
$$

It is assumed that the modal superposition principle is coincident:

$$
p(\sigma, \mathrm{t})=\mathrm{V} \sum_{i} \frac{F_{i}(\sigma) P_{i}(t)}{\left(\omega_{i}^{A}\right)^{2} M_{i}^{A}} \quad(\sigma \in V)
$$

The total non-rigid boundary is defined as two forms, the $D_{B}$ represents the boundary region of the sound absorption, and the $D_{c}$ represents the boundary region of the coupling of the flow and solid.

$$
\begin{aligned}
& \frac{\partial p(\sigma, t)}{\partial \boldsymbol{n}}=-\frac{\rho_{0}}{Z_{B}(\sigma, \mathrm{j} \omega)} \frac{\partial p(\sigma, \mathrm{t})}{\partial t}\left(\sigma \in D_{B}\right) \\
& \frac{\partial p(\sigma, t)}{\partial \boldsymbol{n}}=-\rho_{0} \sum_{j} \psi_{j}(\sigma) q_{j}(t)\left(\sigma \in D_{C}\right)
\end{aligned}
$$

Where $Z_{B}(\sigma, \mathrm{j} \omega)$ is the specific acoustic impedance, $\psi_{j}$ is the inherent vibration of boundary structure, $q_{j}$ is the mode coordinate.

$$
\begin{gathered}
A_{r}=V \sum_{i} \frac{C_{r i} P_{i}}{\left(\omega_{i}^{A}\right)^{2} M_{i}^{A}}+\sum_{j} L_{r j} q_{j} \\
C_{r i}=\iint_{D_{B}} \frac{F_{r} F_{i}}{Z_{B}} d A, \quad L_{r j}=\iint_{D_{C}} F_{r}(\sigma) \psi_{j}(\sigma) d A
\end{gathered}
$$

The acoustic modal equation is obtained:

$$
\begin{aligned}
& \underset{r}{p_{r}}+\left(\omega_{r}^{A}\right)^{2} p_{r}+\sum_{i} \frac{C_{r i} p_{i}}{\left(\omega_{i}^{A}\right)^{2} M_{i}^{A}}=-\frac{1}{V} \sum_{j} L_{r j} q_{j} \\
& (\mathrm{r}=0,1,2, \cdots)
\end{aligned}
$$

\section{The expression of fluid-solid coupling}

Usually when the boundary is thin-walled structures, the case of fluid-solid coupling can be considered, its vibration characteristics are expressed by the linear partial differential equations:

$$
\begin{aligned}
& S \omega(\sigma, t)+m_{C} \partial^{2} \omega(\sigma, t) / \partial^{2} t=p^{C}-p^{E} \\
& \left(\sigma \in D_{C}\right)
\end{aligned}
$$

Where $\mathrm{S}$ is the linear differential operator, $m_{c}$ is the areal density of thin-walled structures, $p^{E}$ and $p^{C}$ are respectively the incentives of the elastic boundary from the external and the inside fluid of cavity. The shape function satisfies the following characteristic equation for linear systems.

$$
S \psi_{j}-m_{C}\left(\omega_{j}^{C}\right)^{2} \psi_{j}=0 \quad(j=1,2, \ldots)
$$

It is obtained from the equation (11) and equation (12):

$$
\sum_{j} m_{C}\left[q_{j}+\left(\omega_{j}^{C}\right)^{2} q_{j}\right] \psi_{j}=p^{C}-p^{E}
$$

According to the orthogonality of shape functions, the modal equation of the boundary is obtained.

$$
M_{j}^{C}\left[q_{j}^{\square}+\left(\omega_{j}^{C}\right)^{2} q_{j}\right]=V \sum_{i} L_{i j} P_{i} /\left[\left(\omega_{i}^{A}\right)^{2} M_{i}^{A}\right]+Q_{j}^{E}
$$

Equation (10) and equation (14) are the mode equations of fluid-solid coupling. If the modes of sound field are taken to the order of $\mathrm{R}+1$, and the structure modes of elastic border are taken to the order of $\mathrm{N}$, it can be expressed to the form of the following matrix equation.

$$
\begin{aligned}
& V\left(\stackrel{\mathbb{P}}{\mathbb{W}} \boldsymbol{M}_{A}^{-1} \stackrel{\square}{\boldsymbol{P}}+\boldsymbol{\Omega}_{A}^{2} \boldsymbol{P}\right)=-\boldsymbol{L} \boldsymbol{q} \\
& \boldsymbol{M}_{C} \stackrel{\boldsymbol{q}}{ }+\boldsymbol{M}_{C} \boldsymbol{\Omega}_{C}^{2} \boldsymbol{q}=V \boldsymbol{L}^{T} \boldsymbol{\Omega}_{A}^{-2} \boldsymbol{M}_{A}^{-1} \boldsymbol{P}+\boldsymbol{Q}_{E}
\end{aligned}
$$

III. THE SOLVING OF THE EQUATIONS OF FLUID-SOLID COUPLING EXCLUDING ABSORPTION BOUNDARY THROUGH THE MODAL METHOD

A. $\quad$ The solving of sound pressure distribution when the elastic boundary is motivated

The elastic boundary is motivated which shows that $p^{E}(\sigma, t) \neq 0$ at the equation (11). 


$$
\begin{aligned}
& {\left[\begin{array}{c}
\mathbb{P} \\
\square \\
\boldsymbol{q}
\end{array}\right]+\left[\begin{array}{cc}
\boldsymbol{\Omega}_{A}^{2}+\boldsymbol{L} \boldsymbol{M}_{C}^{-1} \boldsymbol{L}^{T} \boldsymbol{M}_{A}^{-1} \boldsymbol{\Omega}_{A}^{-2} & -\boldsymbol{L} \boldsymbol{\Omega}_{C}^{2} / V \\
-V \boldsymbol{M}_{C}^{-1} \boldsymbol{L}^{T} \boldsymbol{M}_{A}^{-1} \boldsymbol{\Omega}_{A}^{-2} & \boldsymbol{\Omega}_{C}^{2}
\end{array}\right]\left[\begin{array}{l}
\boldsymbol{P} \\
\boldsymbol{q}
\end{array}\right]=} \\
& {\left[\begin{array}{c}
-\boldsymbol{L} \boldsymbol{M}_{C}^{-1} \boldsymbol{Q}_{E} / V \\
\boldsymbol{M}_{C}^{-1} \boldsymbol{Q}_{E}
\end{array}\right]}
\end{aligned}
$$

In order to express as a standard eigenvalue problem, variable substitution is conducted:

$$
\boldsymbol{p}=\frac{1}{\sqrt{V}} \boldsymbol{\Omega}_{A} \sqrt{\boldsymbol{M}_{A}} \chi_{A} e^{j \omega t}, \boldsymbol{q}=\sqrt{V}\left(\boldsymbol{\Omega}_{C} \sqrt{\boldsymbol{M}_{C}}\right)^{-1} \chi_{C} e^{j \omega t}
$$

The differential equations of decoupling form are obtained by the method of modal superposition:

$$
\begin{aligned}
\boldsymbol{M} \cdot \ddot{\boldsymbol{a}}(t)+\boldsymbol{K} \cdot a(t)=\boldsymbol{T}_{2} \boldsymbol{Q}_{E}(t) \\
\boldsymbol{T}_{2}=\sqrt{V} \boldsymbol{\Omega}^{-2}\left[-\boldsymbol{X}_{A}^{T}\left(\boldsymbol{\Omega}_{A} \sqrt{\boldsymbol{M}_{A}}\right)^{-1} \boldsymbol{L M}_{C}^{-1}+\right. \\
\left.\boldsymbol{X}_{C}^{T}\left(\boldsymbol{\Omega}_{C}^{-1} \sqrt{\boldsymbol{M}_{C}}\right)^{-1}\right]
\end{aligned}
$$

The solution of steady state is obtained for harmonic excitation conditions.

$$
\begin{gathered}
\begin{aligned}
p(\sigma, t)= & \sqrt{V}[\boldsymbol{F}(\sigma)]^{T}\left(\boldsymbol{\Omega}_{A} \sqrt{\boldsymbol{M}_{A}}\right)^{-1} \boldsymbol{X}_{A} \boldsymbol{H}(\omega) \\
& \boldsymbol{T}_{2} \iint_{D_{C}} p^{E}(\sigma) \psi(\sigma) d A \cdot e^{j \omega t}
\end{aligned} \\
\boldsymbol{H}(\omega)=F\{h(t)\}=\int_{-\infty}^{+\infty} h(t) e^{-j \omega t} d t \\
=\left(-\omega^{2} \boldsymbol{I}_{R+N+1}+\Omega^{2}\right)^{-1} M^{-1}
\end{gathered}
$$

\section{B. The analysis and verification of calculation}

According to the established model of sonar cavity and the deduced conclusion of fluid-solid coupling, the parameters of rectangular cavity are set and the boundary conditions is that rectangular plate is four-edge simply supported. It can be analyzed by programming using the MATLAB.

TABLE I. PARAMETERS OF RECTANGULAR CAVITY

\begin{tabular}{|l|c|c|c|}
\hline \multirow{2}{*}{\multicolumn{1}{|c|}{ Item }} & \multicolumn{3}{|c|}{ parameters of rectangular cavity } \\
\cline { 2 - 4 } & Sign & Value & Unit \\
\hline Length & Lx & 0.4 & $\mathrm{~m}$ \\
\hline Width & Ly & 0.6 & $\mathrm{~m}$ \\
\hline Height & $\mathrm{Lz}$ & 0.7 & $\mathrm{~m}$ \\
\hline Density & $\sigma$ & 7800 & $\mathrm{Kg} / \mathrm{m} 3$ \\
\hline Sound Speed & $\mathrm{v}$ & 1500 & $\mathrm{~m} / \mathrm{s}$ \\
\hline
\end{tabular}

TABLE II. PARAMETERS OF RECTANGULAR PLATE

\begin{tabular}{|l|c|c|c|}
\hline \multirow{2}{*}{\multicolumn{1}{|c|}{ Item }} & \multicolumn{3}{|c|}{ parameters of rectangular plate } \\
\cline { 2 - 4 } & Sign & Value & Unit \\
\hline Length & Lx & 0.4 & $\mathrm{~m}$ \\
\hline Width & Ly & 0.6 & $\mathrm{~m}$ \\
\hline Thickness & $\mathrm{h}$ & 0.005 & $\mathrm{~m}$ \\
\hline Density & $\sigma$ & 7800 & $\mathrm{Kg} / \mathrm{m} 3$ \\
\hline Elastic Modulus & $\mathrm{E}$ & $2.0 \mathrm{E}+11$ & $\mathrm{~N} / \mathrm{m} 2$ \\
\hline Poisson ratio & $\mu$ & 0.28 & \\
\hline
\end{tabular}

Fig. 2 and Fig. 3 show that the damping of the cavity wall should be increased at the design of the rectangular cavity and the plate would be thicker under appropriate conditions when the thickness of board is selected. The performance of sound absorption is great and the self-noise is low when the working frequency of the sonar between $700 \mathrm{~Hz}$ and $1400 \mathrm{~Hz}$.

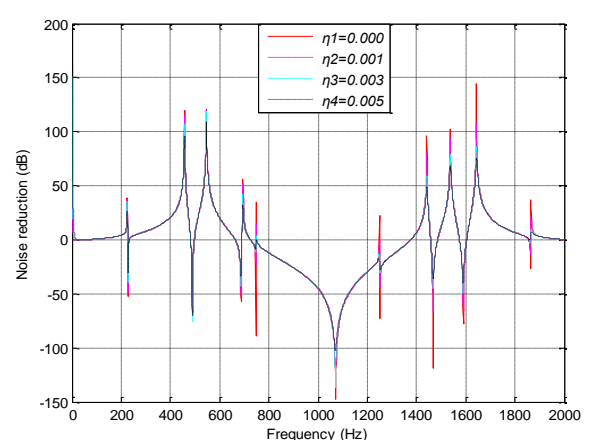

Figure 2. The graph of amount of noise reduction with different damping(45 steel, $\mathrm{h}=5 \mathrm{e}-3 \mathrm{~m})$

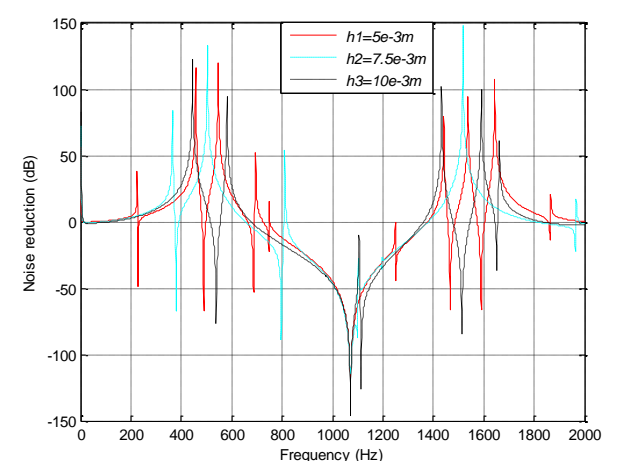

Figure 3. The graph of amount of noise reduction with different damping with different plate thickness

Fig.4 shows that the difference of the same order natural frequency of the coupling is bigger and bigger as the increase of frequency, the size of the cavity has a significant impact on the absorption spectrum of cavity. As shown in Fig.5, the effect of noise reduction is almost unanimously as the change of frequency between the 0 and $100 \mathrm{~Hz}$ at the points $(\mathrm{lx} / 2, \mathrm{ly} / 2, \mathrm{z})$. And the other area that away from the sound source of the cavity has the better noise performance after $1600 \mathrm{~Hz}$. The graph of amount of 
noise reduction is the same at different coordinates on the same height.

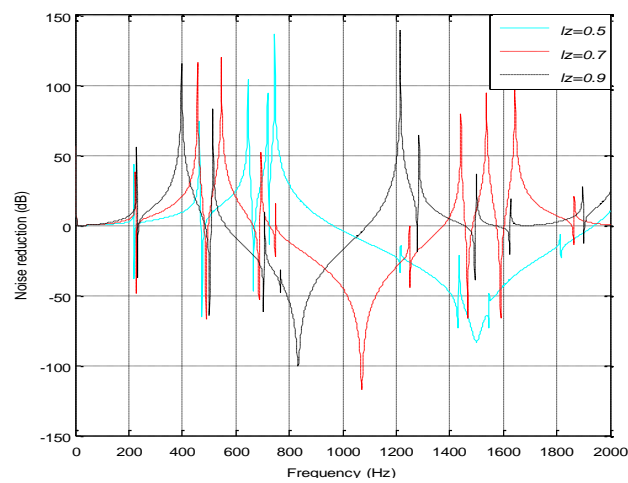

Figure 4. The graph of amount of noise reduction with different heights

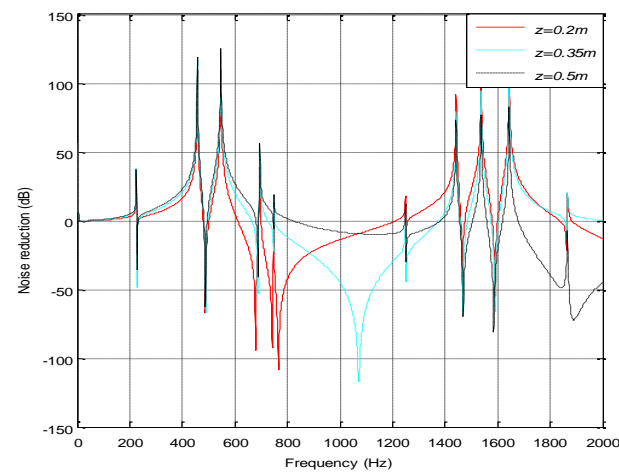

Figure 5. The graph of amount of noise reduction withdifferent coordinate of $\mathrm{z}$

As shown in Fig.6 and Fig.7, the natural frequency of the first order is roughly same as others with same height and different widths of rectangular plate, the natural frequency of the others is lower relatively as the size larger. The natural frequency of the first order is roughly same as others with different size of the cavity; the natural frequency of the others is lower relatively as the size larger and the absorption spectrum of the rectangular plate is the same as others roughly, but the square plate is almost sound-absorbing between $700 \mathrm{~Hz}$ and $2000 \mathrm{~Hz}$.

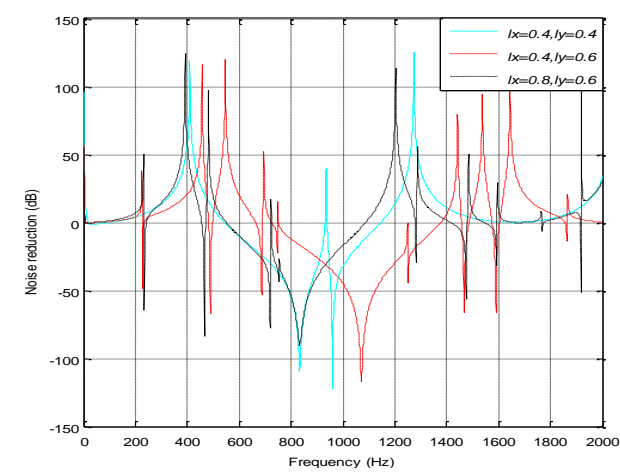

Figure 6. The graph of amount of noise reduction withdifferent length and width

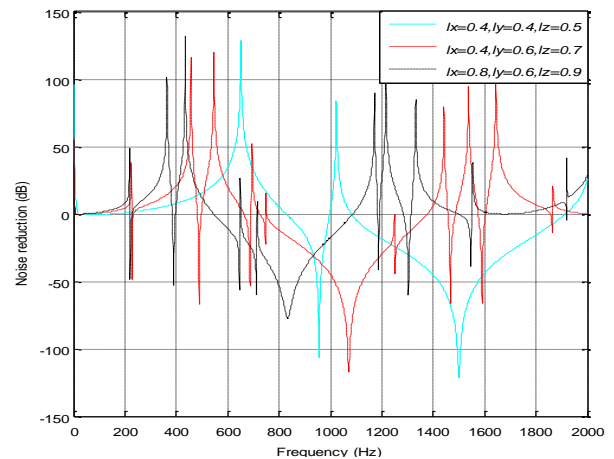

Figure 7. The graph of amount of noise reduction withdifferent length, width and height

As shown in Fig.8 and Fig.9, the amounts of noise reduction at different points in the cavity with the same parameters are analyzed through the MATLAB and Virtual lab respectively. There are similar results at the low frequency compared with the two results; it can be proved that the result of theoretical derivation is receivable considering the error due to the various assumptions.

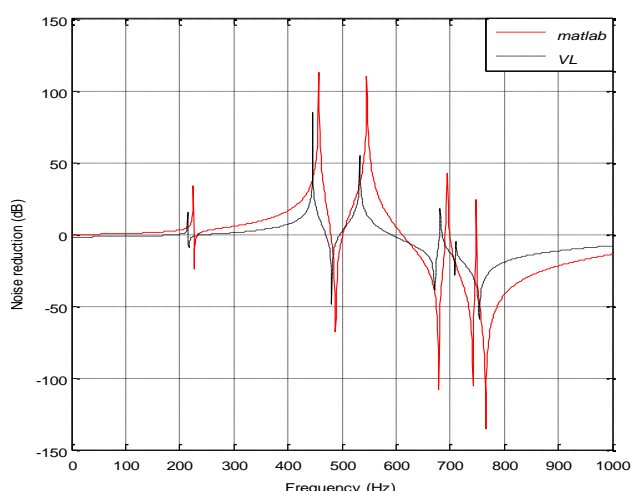

Figure 8 . The graph of the analysis and comparisonat the point of $(\mathrm{Lx} / 2, \mathrm{Ly} / 2,0.2)$

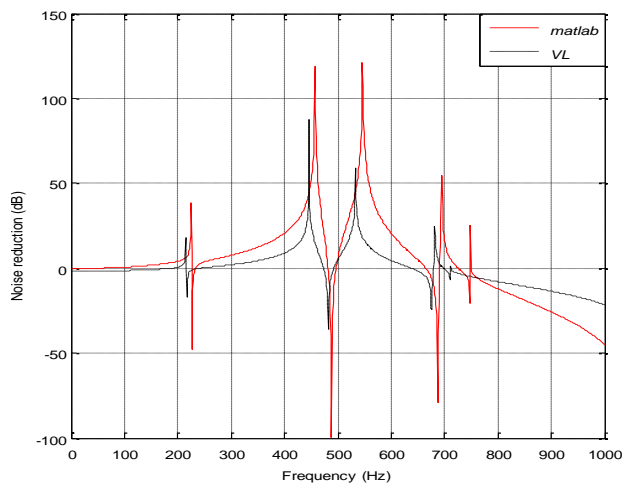

Figure 9. The graph of the analysis and comparisonat the point of $(0.1, \mathrm{Ly} / 2, \mathrm{Lz} / 2)$

\section{CONCLUSION}

The sound pressure of the model of simplified rectangular cavity is deduced considering the fluidstructure coupling effect. It is established of the theoretical 
analysis model of the sonar cavity and the acoustic field in sonar cavity is analyzed theoretically using the modal methods. It is calculated and discussed for the example of the rectangular cavity model; the transmission mechanism of mechanical self-noise is analyzed using the MATLAB through the setting of the rectangular cavity parameter. And the theoretical results are verified through the Virtual lab that is a software about finite element analysis. The influence factors of the sonar self-noise are analyzed and discussed by modifying a series of internal parameters and external dimensions. It can provide certain theoretical basis for the analysis and control of the mechanical self-noise of sonar cavity.

\section{REFERENCES}

[1] Zhang Juan, "Sound Performance Simulation of Typical Structure in Ship," Huazhong University of Science and Technology ,2007, pp. 3-15.

[2] I. S. Jacobs and C. P. Bean, "Fine particles, thin films and exchange anisotropy," in Magnetism, vol. III, G. T. Rado and H. Suhl, Eds. New York: Academic, 1963, pp. 271-350.

[3] Sun Ming, "Research on transmission of Vibretory Noise in Acoustic Covering Layers of Ship," Harbin Engineering University,2010, pp. 4-24.
[4] Oldham D J, Hillarby S N, "The acoustical performance of small close fitting enclosure," Journal of Sound and Vibration, 1991, 150(2): pp.261-281.

[5] Wang Lujun, Ling Qing, Yuan Tingyi, "Sonar Equipment and Technology in America," National defence of Industry Press,2011,pp.15-56.

[6] Ginsberg J H, "Derivation of a Ritz series modeling technique for acoustic cavity-structural systems based on a constrained Hamilton's principle," The Journal of the Acoustical Society of America, 2010, 127(5): pp. 2749-2758.

[7] Ginsberg J H, “On Dowell's simplification for acoustic cavitystructure interaction and consistent alternatives," The Journal of the Acoustical Society of America, 2010, 127(1): pp.22-32.

[8] Yu Mengsa, Liu Yanli, Liao Binbin, "Summarization on the research of coupling character between cavity acoustics and structure vibration together with noise control," Journal of Ship Mechanics,2012,16(1):pp.191-201.

[9] Liu Linxian, "Design of ranging sonar system based on MEMS bionic vector hydrophone," North University of China,2013,pp.523.

[10] Ni Shoulong, Du Liming, Yu Dezhuang, "Effect of key structure parameters for sonar dome on sailing resistance," Ship Science and Technology,2015(1):pp.50-55.

[11] Du Liming, Zhang Jin, "Numerical optimization of sonar dome profile based on reducing its sailing resistance," Ship Science and Technology,2013,35(4):pp.75-79. 